\title{
GENERALIZED TODA BRACKETS AND EQUIVARIANT MOORE SPECTRA
}

\author{
STEVEN R. COSTENOBLE AND STEFAN WANER
}

\begin{abstract}
In this paper we develop a general theory of obstructions to the existence of equivariant Moore spectra. The obstructions we obtain coincide with higher order Toda brackets as defined by Spanier. We then apply the theory to show the existence of equivariant Moore spectra in various special cases.
\end{abstract}

\section{INTRODUCTION}

Steenrod posed the following problem: Given a finite group $G$ and a $\mathbb{Z} G$ module $A$, does there exist a $G$-space $M(A, n)$ whose ordinary nonequivariant homology is $A$, as a $\mathbb{Z} G$-module, concentrated in dimension $n$ ? Carlsson [C1] gave the first counterexample to this general question. On the other hand, sufficient conditions on $A$ and $n$ for the existence of $M(A, n)$ were given by several authors (see for example [K]). Quinn [Q] developed an obstruction theory, as did Smith [S1] later.

The following more general situation seems to have first been considered by Kahn [K]. Let $\mathscr{G}$ be the category whose objects are the $G$-sets $G / H$ and whose morphisms are the $G$-maps. A Bredon system is a contravariant abelian groupvalued functor $T$ on $\mathscr{G}$. A Moore space of type $(T, n)$ is then a based $G$ space $M(T, n)$ such that the Bredon system specified by assigning to $G / K$ the group $\widetilde{H}_{*}\left(M(T, n)^{K} ; \mathbb{Z}\right)$ is isomorphic to $T$ and is concentrated in dimension $n$. This is equivalent to saying that the ordinary reduced equivariant Bredon homology [B] of $M(T, n)$, with coefficients in the constant system $\mathbb{Z}$, is $T$ concentrated in dimension $n$. While Kahn was interested in these spaces for his work in equivariant homology decompositions, they play a fundamental role in equivariant stable homotopy theory when one wishes to endow an arbitrary equivariant cohomology theory with coefficients.

If we are interested in applications to equivariant stable homotopy theory, the question of existence is properly stated as a stable problem, that is, in terms of $G$-spectra. Nonexistence of a Moore space does not imply nonexistence of a corresponding Moore spectrum, so it should be easier to construct Moore spectra. However, in [CW] the authors give an example of a Bredon system for which there is no connective Moore spectrum. Connectivity is a reasonable requirement to impose in order to get a convergent universal coefficients spectral

Received by the editors August 6, 1990.

1991 Mathematics Subject Classification. Primary 55N91; Secondary 55N25, 55P91, 55Q35, 55Q91, 55S36, 55S91. 
sequence. (See $[\mathrm{CW}]$ and also below for further discussion on this point.) In this paper we shall give a theory of obstructions to the existence of connective equivariant Moore spectra, and use this theory to obtain several existence results. Interestingly, the obstructions that emerge turn out to be higher order equivariant Toda brackets, constructed along the lines of Spanier [S2]; see also Cohen [C2].

This paper is organized as follows. In $\S 2$ we develop a general obstruction theory to extending maps in a topological category, the obstructions being welldetermined elements of the groups arising in an associated spectral sequence. In $\S 3$ we adapt arguments of Spanier [S2] to show that the question of the realization of a filtered spectrum, and in particular the question of the existence of an equivariant Moore spectrum, is equivalent to a certain extension problem. In $\S 4$ we apply the results of $\S 2$ to show the existence of well-defined obstructions in the groups of the associated "obstruction spectral sequence." For purposes of computation, we give an alternate (and more classical) description of these obstructions as subsets of the homology of a certain chain complex. In this guise, the obstructions are seen to be higher order equivariant Toda brackets. We apply this obstruction theory in $\S 5$ to give conditions under which the obstruction groups vanish, and we continue in $\S 6$ by listing conditions on the coefficient system $T$ that guarantee the existence of a Moore spectrum of type $T$. An appendix gives some algebraic results used in the rest of the paper.

We devote the rest of this section to stating the existence problem for equivariant Moore spectra precisely. Let $R$ be the regular representation of $G$ and let $\mathscr{U}$ be the orthogonal $G$-vector space $R^{\infty}$. A $G$-universe is an invariant subspace $\mathscr{V}$ of $\mathscr{U}$ that contains a copy of $\mathbb{R}^{\infty}$ with the trivial $G$ action and contains infinitely many copies of every $G$-representation contained in it. As in [LMS], we consider equivariant spectra indexed on invariant subspaces of a given universe $\mathscr{V}$, defined as follows. If $V$ is a $G$-invariant finitedimensional subspace of $\mathscr{V}$, then denote its one-point compactification by $S^{V}$. A $\mathscr{V}$-spectrum is a collection of based $G$-spaces $E(V)$, indexed on the finitedimensional invariant subspaces of $\mathscr{V}$, together with $G$-maps

$$
\sigma: \Sigma^{V} E(W) \rightarrow E(V+W) \text { for } V \perp W
$$

where $\Sigma^{V} X=X \wedge S^{V}$, such that $\sigma=1$ when $V=\{0\}$, and the diagram

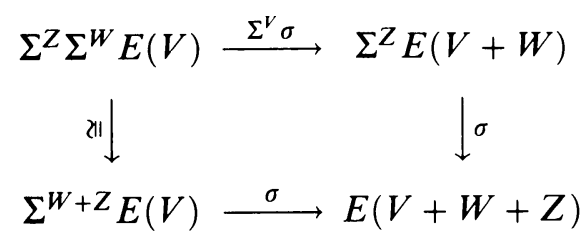

commutes when $V, W$, and $Z$ are pairwise orthogonal. Further, one requires that the maps $\tilde{\sigma}: E(W) \rightarrow \Omega^{V} E(V+W)$, the adjoints of the structure maps $\sigma$, are $G$-homeomorphisms. We shall refer to a $\mathscr{V}$-spectrum as a Bredon spectrum if $\mathscr{V}=\mathbb{R}^{\infty}$, and a Mackey spectrum if $\mathscr{V}=\mathscr{U}$, and shall restrict attention in this paper to these two cases.

Before we can say what equivariant Moore spectra are, we need something more general than the "Bredon systems" defined above. If $\mathscr{V}$ is a $G$-universe as above, let $\mathscr{O}_{G}(\mathscr{V})$ be the category whose objects are the $G$-orbits $G / H$ and 
whose morphisms are the $\mathscr{V}$-stable maps, given by

$$
\mathscr{O}_{G}(\mathscr{V})(G / H, G / K)=\underset{V \subset \mathscr{V}}{\operatorname{colim}}\left[\Sigma^{V} G / H^{+}, \Sigma^{V} G / K^{+}\right]_{G},
$$

where the ${ }^{+}$denotes addition of disjoint basepoints. $\mathscr{O}_{G}(\mathscr{V})(G / H, G / K)$ may be equivalently defined as $G$-homotopy classes of maps of $\mathscr{V}$-spectra $\Sigma^{\infty} G / H^{+}$ $\rightarrow \Sigma^{\infty} G / K^{+}$, where $\Sigma^{\infty}$ converts based $G$-spaces to $G$-spectra and is left adjoint to the zeroth space functor $\{E(V)\} \mapsto E(0)$. An $\mathscr{O}_{G}(\mathscr{V})$-functor is a contravariant additive abelian group-valued functor on $\mathscr{O}_{G}(\mathscr{V})$. We refer to an $\mathscr{O}_{G}(\mathscr{V})$-functor as a Mackey functor when $\mathscr{V}=\mathscr{U}$, and as a Bredon functor when $\mathscr{V}=\mathbb{R}^{\infty}$. (See [W] for a discussion of such functors.)

As outlined in [LMM], there is an ordinary cohomology theory defined on $\mathscr{V}$-spectra, with coefficients in any $\mathscr{O}_{G}(\mathscr{V})$-functor. This theory is represented in the category of $\mathscr{V}$-spectra. Denote by $A_{G}$ the canonical projective $\mathscr{O}_{G}(\mathscr{V})$ functor $G / H \mapsto \mathscr{O}_{G}(\mathscr{V})(G / H, G / G)$, and denote by $H_{*}^{G}\left(-; A_{G}\right)$ the represented homology theory dual to the ordinary cohomology theory $H_{G}^{*}\left(-; A_{G}\right)$. The assignment $G / J \mapsto H_{r}^{J}\left(-; A_{J}\right)$ is then an $\mathscr{O}_{G}(\mathscr{V})$-functor which we denote by $\underline{H}_{r}\left(-; A_{G}\right)$. If $T$ is an $\mathscr{O}_{G}(\mathscr{V})$-functor, define an equivariant Moore spectrum of type $T$ to be a $\mathscr{V}$-spestrum $M T$ with

$$
\underline{H}_{r}\left(M T ; A_{G}\right)= \begin{cases}T & \text { if } r=0 ; \\ 0 & \text { if } r \neq 0 .\end{cases}
$$

In the case $\mathscr{V}=\mathbb{R}^{\infty}, \underline{H}_{*}\left(M T ; A_{G}\right)(G / K)=H_{*}\left(M T^{K} ; \mathbb{Z}\right)$, so that our definition is a direct generalization of Kahn's [K]. In both of the cases $\mathscr{V}=\mathbb{R}^{\infty}$ and $\mathscr{V}=\mathscr{U}$, we have

$$
\left(H A_{G} \wedge M T\right)^{*}(E) \cong H_{G}^{*}(E ; T)
$$

for any $\mathscr{V}$-spectrum $E$, where $H A_{G}$ is the Eilenberg-Mac Lane spectrum representing ordinary cohomology with coefficients in $A_{G}$. As alluded to above, we also insist that $M T$ be connective and $\mathrm{CW}$. These extra conditions are needed to give us a convergent universal coefficients spectral sequence: First, recall that if $X$ is a $G$-CW spectrum, with skeleta $X_{n}$, then the cellular chain complex of $X$ is the complex of $\mathscr{O}_{G}(\mathscr{V})$-functors $C_{n} X$ given by $C_{n} X(G / H)=$ $\left[\Sigma^{n} \Sigma^{\infty} G / H^{+}, X_{n} / X_{n-1}\right]_{G}$. Observe that the cellular chains of $M T$ give a resolution of $T$ in the category of $\mathscr{O}_{G}(\mathscr{V})$-functors. Indeed, this follows from the fact that, since $\mathscr{V}=\mathscr{U}$ or $\mathbb{R}^{\infty}, \underline{H}_{r}\left(-; A_{G}\right)$ is an ordinary equivariant homology theory in that it satisfies a dimension axiom in the sense of Bredon [B], and is thus computable as the homology of the cellular chain complex. Now if $E$ is a $\mathscr{V}$-spectrum with associated generalized $G$-cohomology theory $E_{G}^{*}(-)$ and $\mathscr{O}_{G}(\mathscr{V})$-functor $\underline{E}^{*}(-)$, then a cellular filtration of $M T$ gives, in the usual way, a universal coefficients spectral sequence converging from $\operatorname{Ext}^{*}\left(\underline{E}^{*}(-), T\right)$ to $(\underline{E \wedge M T})^{*}(-)$, Ext being taken in the category of $\mathscr{O}_{G}(\mathscr{V})$-functors.

\section{OBSTRUCTION THEORY}

We develop a theory of obstructions, generalizing classical obstruction theory, to extending maps in a topological category $\mathscr{C}$. We begin with the usual extension problem: given $l: A \rightarrow X$ and $f: A \rightarrow Y$ in $\mathscr{C}$, what are the obstructions to factoring $f$ through $X$ up to homotopy? Here, two maps $A \rightarrow Y$ are homotopic if they are in the same path component of the function space $\mathscr{C}(A, Y)$. 
As in the classical theory, we assume given a filtration of $l$, which is a sequence

$$
A=X_{-1} \rightarrow X_{0} \rightarrow X_{1} \rightarrow \cdots \rightarrow X_{n} \rightarrow \cdots \rightarrow X
$$

such that each map $X_{r} \rightarrow X_{r+1}$ is a cofibration and $X$ is the categorical colimit of the $X_{r}$ 's. Precisely, we require that, for any object $Y$, the restrictions $\mathscr{C}\left(X_{r+1}, Y\right) \rightarrow \mathscr{C}\left(X_{r}, Y\right)$ be fibrations, and that $\mathscr{C}(X, Y)$ be homeomorphic to $\lim _{r} \mathscr{C}\left(X_{r}, Y\right)$. If we write $[X, Y]$ for the set of homotopy classes of maps, or $\pi_{0} \mathscr{C}(X, Y)$, this implies that $[X, Y] \rightarrow \lim \left[X_{r}, Y\right]$ is onto.

If $Z$ is a based space let $\omega_{*}(Z)$ denote its stable homotopy groups; if $F$ is an unbased space let $F^{+}$denote $F$ with a disjoint basepoint adjoined. Observe that, since $\omega_{0}\left(\mathscr{C}(X, Y)^{+}\right)$is the free abelian group on $[X, Y]$, the given element $[f]$ of $[A, Y]$ is in the image of $[X, Y]$ under $l^{*}$ iff $[f] \in$ $l^{*} \omega_{0}\left(\mathscr{C}(X, Y)^{+}\right) \subset \omega_{0}\left(\mathscr{C}(A, Y)^{+}\right)$. We therefore consider the exact couple

$$
\omega_{-p-q}\left(\mathscr{C}\left(X_{p}, Y\right)^{+}\right) \longrightarrow \omega_{-p-q}\left(\mathscr{C}\left(X_{p-1}, Y\right)^{+}\right)
$$

where $C \sigma_{p}$ is the cofiber of $\sigma_{p}: \mathscr{C}\left(X_{p}, Y\right) \rightarrow \mathscr{C}\left(X_{p-1}, Y\right)$. Writing the diagram as

$$
D_{k_{1}}^{p, q} \underset{E_{1}^{p, q+1}}{\stackrel{i_{1}}{\swarrow j_{1}}} D_{1}^{p-1, q+1}
$$

we see that the obstruction to extending $f$ over $X_{0}$ is $\theta_{0}=j_{1}[f] \in E_{1}^{0,1}$. If this vanishes, then $[f] \in i_{1}\left(D_{1}^{0,0}\right)=D_{2}^{-1,1}$, where $D_{2}^{*}$ is the corresponding term in the derived couple. For $f$ to extend over $X_{1},[f]$ must be in $\operatorname{Im} i_{1}^{2}=\operatorname{Im} i_{2}=\operatorname{Ker} j_{2}$, so that the obstruction to extending $f$ over $X_{1}$ is $\theta_{1}=j_{2}[f] \in E_{2}^{1,0}$. Continuing inductively, we see that, if $f$ extends over $X_{r-1}$, then the obstruction to constructing an extension of $f$ over $X_{r}$ is $\theta_{r}=j_{r+1}[f] \in E_{r+1}^{r,-r+1}$.

If all the obstructions $\theta_{r}(r \geq 0)$ vanish, then there exists an element of $\lim \left[X_{r}, Y\right]$ restricting to $[f] \in\left[\bar{X}_{-1}, Y\right]$. By our assumption on the filtration, this is equivalent to saying that there is an element of $[X, Y]$ that restricts to $[f]$. We thus conclude

Theorem 2.1. If $\left\{X_{r}\right\}$ is a filtration of $l: A \rightarrow X$ and if $f: A \rightarrow Y$ is any map, then $f$ is homotopic to a map that extends over $X$ iff all of the obstructions $\theta_{r} \in E_{r+1}^{r,-r+1}$ vanish.

In view of Theorem 2.1, we shall refer to the spectral sequence $E_{*}^{*, *}$ as the extension spectral sequence. Notice that $E_{r}^{p, q}=0$ if $p+q>1$ or if $p<0$. Therefore, most of the spectral sequence lies in a wedge in the fourth quadrant.

Classically, obstructions are viewed as subsets of certain homology groups. The following examples show the relationship between these obstruction subsets and our obstruction elements.

Example 2.3. (a) Extension of maps of spectra. Let $A$ be a sub-CW-spectrum of the CW-spectrum $X$, assume that $X / A$ is connective, and let $f: A \rightarrow Y$ be a map of spectra. If $\left\{X_{n}\right\}$ is the standard relative skeletal filtration, then the 
associated exact couple

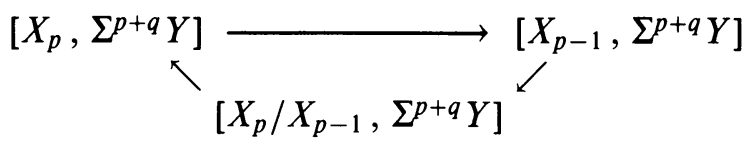

gives rise to the Atiyah-Hirzebruch spectral sequence

$$
A_{2}^{p, q}=H^{p}\left(X, A ; \pi_{-q}(Y)\right) \Rightarrow\left[X / A, \Sigma^{p+q} Y\right] .
$$

By an argument similar to that given above, the obstructions to a homotopy extension of $f$ over $X$ are well-defined elements $\mu_{r} \in A_{r+1}^{r,-r+1}$, these groups being subquotients of $H^{r}\left(X, A ; \pi_{r-1}(Y)\right)$ for $r \geq 2$. The corresponding coset in $H^{r}\left(X, A ; \pi_{r-1}(Y)\right)$ coincides with the classical obstruction, which is defined as a subset of this group. Further, the extension spectral sequence maps into the Atiyah-Hirzebruch spectral sequence through a map of exact couples, given by the composites

$$
\omega_{-p-q}\left(F\left(X_{p}, Y\right)^{+}\right) \rightarrow \omega_{-p-q}\left(F\left(X_{p}, Y\right)\right) \rightarrow\left[X_{p}, \Sigma^{p+q} Y\right]
$$

and

$$
\omega_{-p-q+1}\left(C \sigma_{q}\right) \stackrel{\nu}{\rightarrow} \omega_{-p-q+1}\left(F\left(\Sigma^{-1} X_{p} / X_{p-1}, Y\right)\right) \rightarrow\left[X_{p} / X_{p-1}, \Sigma^{p+q} Y\right] .
$$

Here, $\nu$ is induced by the map $C \sigma_{p} \rightarrow F\left(\Sigma^{-1} X_{p} / X_{p-1}, Y\right)$ that results from the fact that $F\left(X_{p}, Y\right)$ is equivalent to the fiber of

$$
F\left(X_{p-1}, Y\right) \rightarrow F\left(\Sigma^{-1} X_{p} / X_{p-1}, Y\right) .
$$

Under this map, $\theta_{r}$ maps to $\mu_{r}$.

(b) Extension of maps and spaces. Let $A$ be a subcomplex of the based CW-complex $X$, and let $f: A \rightarrow Y$ be a based map of spaces. If $\left\{X_{n}\right\}$ is the standard relative skeletal filtation, and if $X_{n}$ is the cofiber of the attaching map $J_{n-1} \rightarrow X_{n-1}\left(J_{n-1}\right.$ being a wedge of $(n-1)$-spheres $)$, then we have an exact sequence of sets

$$
\cdots \rightarrow\left[X_{p}, Y\right] \rightarrow\left[X_{p-1}, Y\right] \rightarrow\left[J_{p-1}, Y\right]=C^{p}\left(X, A ; \pi_{p-1}(Y)\right) .
$$

The classical obstruction to extending $f$ over the $r$-skeleton is then a subset $\mu_{r}$ of the cocycles in $C^{r}\left(X, A ; \pi_{r-1}(Y)\right)$, and is usually viewed as a subset of homology. In order to compare these obstructions with the $\theta_{r}$, we first stabilize the above sequence to obtain

$$
\cdots \rightarrow \omega_{0}\left(F\left(X_{p}, Y\right)^{+}\right) \rightarrow \omega_{0}\left(F\left(X_{p-1}, Y\right)^{+}\right) \rightarrow \omega_{0}\left(F\left(J_{p-1}, Y\right)\right) .
$$

As in (a), there is a map $C \sigma_{p} \rightarrow F\left(J_{p-1}, Y\right)$, and the obstruction $\theta_{r}$, considered as a subset of $E_{1}^{r,-r+1}$, is taken by this map into a coset containing the image of $\mu_{r}$ in $\omega_{0}\left(F\left(J_{r-1}, Y\right)\right)$ under stabilization.

\section{REALIZING A FILTERED SPECTRUM}

Although the discussion in this section is concerned with equivariant spectra, our results remain pertinent even if the group is trivial. Let $X$ be a $G$-spectrum indexed on any $G$-universe. A filtration of $X$ is a sequence $\left\{X_{n}\right\}_{n>0}$ of subspectra of $X$ with $X_{n} \subset X_{n+1}$ such that $X=\operatorname{colim}_{n}\left(X_{n}\right)$. (See [LMS] for 
details regarding the category of $G$-spectra.) Let $W_{n}=\Sigma^{-n} X_{n} / X_{n-1}$. One may now construct the following diagram.

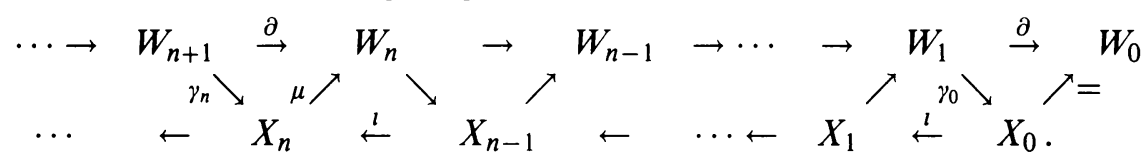

Here, the triangles with base $l$ are cofiber triangles and the maps $\partial$ are defined as the composites $\mu \circ \gamma$. The degrees of the various maps are thus given by $\operatorname{deg}(\partial)=\operatorname{deg}(l)=0$ and $\operatorname{deg}\left(\gamma_{n}\right)=n=-\operatorname{deg}\left(\mu: X_{n} \rightarrow W_{n}\right)$.

Conversely, given a sequence $\partial: Z_{n+1} \rightarrow Z_{n}$ of maps of $G$-spectra, one may ask whether there exists a filtered $G$-spectrum $X$ with homotopy equivalences $\Sigma^{-n} X_{n} / X_{n-1} \simeq Z_{n}$ making the following diagrams $G$-homotopy commute:

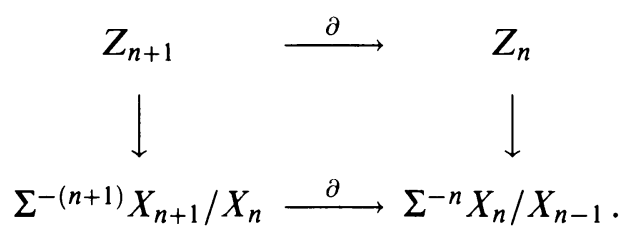

If such an $X$ exists, we call $X$ a realization of the sequence $\left\{Z_{n} ; \partial\right\}$.

In [S2], Spanier considered sequences of maps of spaces and defined higher order Toda brackets as the obstructions to solving an associated extension problem. Based on his techniques, we now convert our realization problem into an extension problem.

If $\Gamma$ is a topological space, recall from [M] that a topological graph over $\Gamma$ is just a space $A$ with source and target maps $S, T: A \rightarrow \Gamma$. Maps of topological graphs over $\Gamma$ are continuous maps preserving source and target. A topological composition graph over $\Gamma$ is a semigroup in the category of topological graphs over $\Gamma$; that is, defining

$$
A \times_{\Gamma} A=\{(f, g) \mid S(f)=T(g)\},
$$

$S(f, g)=S(g)$ and $T(f, g)=T(f)$, we are given a composition map $c: A \times_{\Gamma}$ $A \rightarrow A$ preserving source and target and satisfying the usual associativity axiom. In other words, this is a topological category with object space $\Gamma$ but without identity maps. We will write $A(\alpha, \beta)$ for the set of elements with source $\alpha$ and target $\beta$, and call these the arrows from $\alpha$ to $\beta$. Morphisms between two composition graphs over $\Gamma$ are again given by continuous maps between the arrow spaces, but now they are required to preserve composition as well as source and target. We will write $\mathscr{F}(A, B)$ for the space of maps over $\Gamma$ from $A$ to $B$, topologized as a subspace of the usual space of continuous functions between the arrow spaces. This makes the collection of composition graphs over $\Gamma$ a topological category. Homotopies in this category are defined as one-parameter families of maps, as at the beginning of $\S 2$.

We shall be working with the following topological composition graphs over $\mathbb{N}=\{0,1,2, \ldots\}$. First define $\square$ to have arrows $\square(m, n)=I^{(m-n-1)}$ when $m>n$, where $I^{(r)}$ denotes the $r$-fold smash product $I \wedge I \wedge \cdots \wedge I$ with $I$ based at 1 , and where $I^{(0)}=\{\underline{0}, \underline{1}\}$. When $m \leq n$, we take $\square(m, n)=\varnothing$. Composition in $\square$ is given by

$$
\square(n, r) \times \square(m, n)=I^{(n-r-1)} \times I^{(m-n-1)} \stackrel{\zeta}{\rightarrow} I^{(r-m-1)}=\square(m, r),
$$

where $\zeta(x, y)=(x, 0, y)$. 
If $Z=\left(Z_{n}\right)_{n \geq 0}$ is any sequence of $G$-spectra, define an associated topological composition graph $\Phi(Z)$ over $\mathbb{N}$ by taking $\Phi(Z)(m, n)=F^{G}\left(Z_{m}, Z_{n}\right)$, the space of maps of $G$-spectra $Z_{m} \rightarrow Z_{n}$, and where composition is just composition in the category of $G$-spectra.

Define a subgraph $\square_{0}$ of $\square$ by taking $\square_{0}(m, n)=S^{0}$ for $m>n$, where we take $S^{0}=\{(0,0, \ldots, 0), *\} \subset \square(m, n)$. Given a sequence $\left\{\partial: Z_{n+1} \rightarrow Z_{n}\right\}$ of maps of $G$-spectra, define a map of composition graphs $f_{Z}: \square_{0} \rightarrow \Phi(Z)$ by sending $(0,0, \ldots, 0) \in \square_{0}(m, n)$ to $\partial^{m-n}: Z_{m} \rightarrow Z_{n}$ and $*$ to the trivial map.

Theorem 3.1. The sequence $\partial: Z_{n+1} \rightarrow Z_{n}$ of maps of $G$-spectra can be realized as the filtration quotients of a filtered spectrum iff $f_{Z}: \square_{0} \rightarrow \Phi(Z)$ is homotopic to a map that extends to

We first prove a lemma that is a variant of [S2, Theorem 6.1]. Suppose that $B=\left\{\beta_{n}: B_{n} \rightarrow B_{n-1}\right\}$ is a sequence of maps with the property that $\beta_{1} \circ \beta_{2} \simeq 0$ through a specified null-homotopy $H$ of $G$-spectra. Then $H$ determines a map $\gamma: B_{2} \rightarrow F \beta_{1}$. Denote the sequence $\left\{\gamma, \beta_{3}, \beta_{4}, \ldots\right\}$ by $B^{\prime}$.

Lemma 3.2. Extensions to $\square$ of $f_{B}$ which restrict to $H$ on $\square(2,0)$ are in one-to-one correspondence with extensions of $f_{B^{\prime}}$ to $\square$.

Proof. Let $\tilde{f}_{B}$ be an extension of $f_{B}$ to $\square$ which restricts to $H$ on $\square(2,0)$. We define an associated extension of $f_{B^{\prime}}$ by giving the maps $\square(m, n) \rightarrow$ $\Phi\left(B^{\prime}\right)(m, n)$. We consider two cases. If $n \neq 0$, then define $\tilde{f}_{B^{\prime}}(x: m \rightarrow n)=$ $\tilde{f}_{B}(x: m+1 \rightarrow n+1)$. If $n=0$ then we must define a map $\tilde{f}_{B^{\prime}}: \square(m, 0) \rightarrow$ $F^{G}\left(B_{m+1}, F \beta_{1}\right)$. Now a $G$-map $B_{m+1} \rightarrow F \beta_{1}$ corresponds to a pair $(\phi, \lambda)$ where $\phi$ is a $G$-map $B_{m+1} \rightarrow B_{1}$ and $\lambda$ is a null-homotopy of $\beta_{1} \circ \phi$. Such pairs are described by $\left(\tilde{f}_{B}(x: m+1 \rightarrow 1), \tilde{f}_{B}((\underline{0}: 1 \rightarrow 0) \circ x)\right)$.

Conversely, given $\tilde{f}_{B^{\prime}}$, we can recover $\tilde{f}_{B}$ using the same correspondence between maps into a fiber and pairs of maps.

Proof of Theorem 3.1. Given an extension to $\square$ of a map homotopic to $f_{Z}$, we can apply the lemma inductively to obtain a diagram

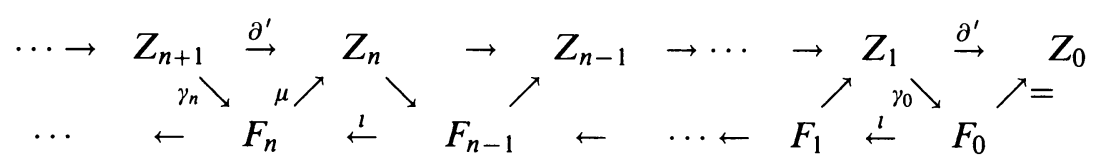

where the maps $\partial^{\prime}$ are $G$-homotopic to the original maps $\partial$ and $F_{n}=F \gamma_{n-1}$. Up to homotopy equivalence, we can replace fibers by cofibers, thereby obtaining a realization of $Z$.

Conversely, given a realization of $Z$, we can construct a diagram as above. Given the diagram, we can now define an extension $\tilde{f}_{Z}$ of a homotope of $f_{Z}$ as follows. Consider the diagram

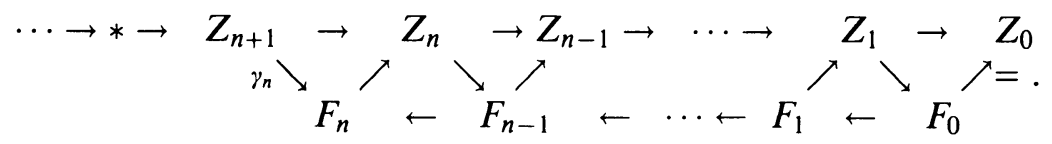

We can easily solve the extension problem for the sequence $\left\{\gamma_{n}, *, *, \ldots\right\}$, $\Phi\left\{\gamma_{n}, *, *, \ldots\right\}$ being trivial. It follows inductively by the lemma that this 
gives a unique extension $\tilde{f}_{Z}^{n}$ for the sequence $\left(\partial_{1}^{\prime}, \ldots, \partial_{n+1}^{\prime}, *, *, \ldots\right)$. Moreover, the construction in that lemma shows that $\tilde{f}_{Z}^{n}=\tilde{f}_{Z}^{n-1}$ on the full subgraph of $\square$ with objects $\{0, \ldots, n\}$. This tells us how to define $\tilde{f}_{Z}$ on all of $\square$.

\section{THE OBSTRUCTIONS TO REALIZING A FILTERED SPECTRUM}

In order to apply the obstruction theory in $\S 2$ to the extension problem of $\S 3$, we filter the graph $\square$ by defining $\square_{p}$ to be the subgraph over $\mathbb{N}$ of $\square$ generated by all of the arrows $m \rightarrow n$ where $m>n \geq m-p-1$. The $E_{1}$-level of the extension spectral sequence is then given by the exact couple

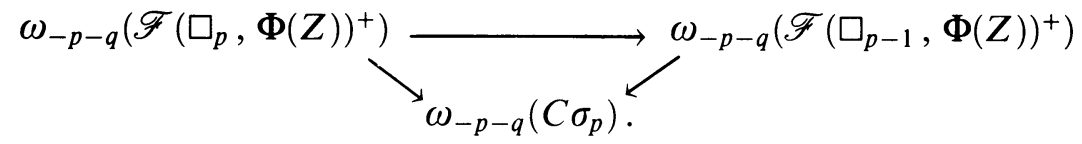

Notice that the first obstruction, the obstruction to extending over $\square_{1}$, is simply the collection of maps $\partial \circ \partial$. Thus the first obstruction is just the obvious one that the composites $\partial^{2}$ should be null-homotopic. The first interesting obstruction then is usually the next.

This approach makes the obstructions to realizing a filtered spectrum welldefined elements of rather complicated groups. For practical purposes, it will prove useful to realize these obstructions as somewhat complicated subsets of more tractible groups. Further, these new obstruction subsets will be seen to be generalizations of Spanier's higher order Toda brackets [S2, C2].

Start with the exact sequence

$$
\left[\square_{p}, \Phi\right] \rightarrow\left[\square_{p-1}, \Phi\right] \rightarrow\left[J_{p-1}, \Phi\right] .
$$

Here, $J_{p-1}$ is the subcomposition graph of $\square_{p-1}$ generated by the morphisms in $\square_{p-1}(n, n-p-1)=\partial I^{(p)} \subset I^{(p)}=\square(n, n-p-1)$, for all $n$. By the usual argument, the obstruction to extending $f_{Z}$ over $\square_{p}$ is a subset $\mu_{p}$ of $\left[J_{p-1}, \Phi\right]$. Since $J_{p-1}$ is generated by its arrow spaces $J_{p-1}(n, n-p-1)$, it follows that

$$
\left[J_{p-1}, \Phi\right] \cong \prod_{n}\left[\Sigma^{p-1} Z_{n}, Z_{n-p-1}\right]_{G},
$$

where $[-,-]_{G}$ denotes homotopy classes of $G$-maps between $G$-spectra. Thus the obstruction $\mu_{p}$ is a subset of this product, and $f_{Z}$ extends over $\square_{p}$ if and only if this subset contains 0 .

If we start with a finite sequence of based spaces $\cdots \rightarrow * \rightarrow Z_{n} \rightarrow \cdots \rightarrow$ $Z_{0}$, then our obstruction $\mu_{n-1}$ coincides with Spanier's $n$-fold Toda bracket $[\mathrm{S} 2, \S 6]$. (Note that in this special case we have $\left[J_{n-2}, \Phi\right] \cong\left[\Sigma^{n-2} Z_{n}, Z_{0}\right]_{G}$, since the other factors are trivial.) It follows that we can think of the $\mu_{n}$ as generalized higher order Toda brackets.

The map $d=\partial^{*}+(-1)^{q} \partial_{*}: \prod_{n}\left[\Sigma^{p} Z_{n}, Z_{n-q-1}\right]_{G} \rightarrow \prod_{n}\left[\Sigma^{p} Z_{n}, Z_{n-q-2}\right]_{G}$, induced by composition on either size with $\partial$, is a differential. If we let ${ }_{p} C^{q}=$ $\prod_{n}\left[\Sigma^{p} Z_{n}, Z_{n-q-1}\right]_{G}$, then we get a cochain complex $\left({ }_{p} C^{*}, d\right)$ for each $p$. We now have the following.

Lemma 4.1. The obstruction subset $\mu_{p}$ consists of cocycles in ${ }_{p-1} C^{p}$.

Proof. First, note that

$$
\begin{aligned}
{\left[\Sigma^{p-1} Z_{n}, Z_{n-p-1}\right]_{G} } & \cong \operatorname{Hom}\left(\mathbb{Z}, \pi_{p-1}(\Phi(n, n-p-1))\right) \\
& \cong \operatorname{Hom}\left(\pi_{p-1}\left(J_{p-1}(n, n-p-1)\right), \pi_{p-1}(\Phi(n, n-p-1))\right) .
\end{aligned}
$$


Let $K_{p-1}(n, n-p-2) \subset \square_{p-1}(n, n-p-2)$ be the two faces given by the image of $\square_{p-1}(n, n-p-1) \cup \square_{p-1}(n-1, n-p-2)$ under composition with the maps $\underline{0}: n-p-1 \rightarrow n-p-2$ and $n \rightarrow n-1$. These spaces are part of a cofibration sequence of spaces

$$
\begin{aligned}
K_{p-1}(n, n-p-2) & \rightarrow \square_{p-1}(n, n-p-2) \rightarrow \square_{p}(n, n-p-2) \\
& \rightarrow \Sigma K_{p-1}(n, n-p-2) \rightarrow \cdots .
\end{aligned}
$$

Given a map $h: J_{p-1} \rightarrow \Phi$ which extends to $\square_{p-1}$, we have maps

$$
h_{n}: \pi_{p-1}\left(J_{p-1}(n, n-p-1)\right) \rightarrow \pi_{p-1}(\Phi(n, n-p-1)),
$$

and we can describe the components of $d\left(h_{n}\right)$ as the composites

$$
\begin{aligned}
\pi_{p}\left(J_{p}(n, n-p-2)\right) & \stackrel{\equiv}{\rightarrow} \pi_{p}\left(\square_{p}(n, n-p-2)\right) \rightarrow \pi_{p}\left(\Sigma K_{p-1}(n, n-p-2)\right) \\
& \cong \pi_{p-1}\left(K_{p-1}(n, n-p-2)\right) \rightarrow \pi_{p-1}\left(\square_{p-1}(n, n-p-2)\right) \\
& \rightarrow \pi_{p-1}(\Phi(n, n-p-2)) .
\end{aligned}
$$

To see that this is indeed $d\left(h_{n}\right)$, note that the composite

$\pi_{p-1}\left(K_{p-1}(n, n-p-2)\right) \rightarrow \pi_{p-1}\left(\square_{p-1}(n, n-p-2)\right) \rightarrow \pi_{p-1}(\Phi(n, n-p-2))$

is $(-1)^{n} \partial_{*} h_{n}$ on the image of $J_{p-1}(n, n-p-1)=\square_{p-1}(n, n-p-1)$, and $\partial^{*} h_{n-1}$ on the image of $J_{p-1}(n-1, n-p-2)=\square_{p-1}(n-1, n-p-2)$.

On the other hand, the composite from $\pi_{p}\left(\square_{p}(n, n-p-2)\right)$ to

$$
\pi_{p-1}\left(\square_{p-1}(n, n-p-2)\right)
$$

coincides with the composite

$$
\begin{aligned}
\pi_{p}\left(\square_{p}(n, n-p-2)\right) & \rightarrow \pi_{p}\left(\square_{p}(n, n-p-2), \square_{p-1}(n, n-p-2)\right) \\
& \rightarrow \pi_{p-1}\left(\square_{p-1}(n, n-p-2)\right)
\end{aligned}
$$

and is thus zero.

Lemma 4.2. If $p \geq 2$, then $\mu_{p}$ is a union of cosets of the image of $d$.

Proof. $\mu_{p}$ is the image in $\left[J_{p-1}, \Phi\right]$ of all possible homotopy lifts of $f_{Z}$ to $\square_{p-1}$. Consider one such lift $f_{p-1}$, and its restriction $f_{p-2}$ to $\square_{p-2}$. We can alter $f_{p-1}$ using any element $g$ of $\prod_{n}\left[\Sigma^{p-1} Z_{n}, Z_{n-p-2}\right]_{G}$ by considering the latter as $\prod_{n}\left[S^{p-1}, F\left(Z_{n}, Z_{n-p-2}\right)\right]_{G}$. The effect of this on the obstruction is to change it by $d(g)$.

In view of Lemmas 4.1 and 4.2 , we may regard the $p$ th obstruction $\mu_{p}$ as a subset of $H^{p}\left({ }_{p-1} C^{*}, d\right)$ when $p \geq 2$.

\section{Equivariant CONNECTIVE MoORE SPECTRA}

Let $T$ be an $\mathscr{O}_{G}(\mathscr{V})$-functor, and let $\left\{P_{*}, \partial\right\}$ be a projective resolution of $T$, where each $P_{i}$ is a direct sum of canonical projectives of the form $\mathscr{O}_{G}(\mathscr{V})(-, G / H)$ for various $H$. We construct a tower of $\mathscr{V}$-spectra $W=$ $\left\{W_{i}, \partial_{i}\right\}$ as follows. First, let $W_{i}$ have one wedge summand $\Sigma^{\infty} G / H^{+}$for each direct summand $\mathscr{O}_{G}(\mathscr{V})(-, G / H)$ of $P_{i}$. The boundary maps $W_{i} \rightarrow W_{i-1}$ are then specified (up to homotopy) using

$$
\begin{aligned}
{\left[\Sigma^{\infty} G / H^{+}, \Sigma^{\infty} G / K^{+}\right]_{G} } & =\mathscr{O}_{G}(\mathscr{V})(G / H, G / K) \\
& \cong \operatorname{Hom}\left(\mathscr{O}_{G}(\mathscr{V})(-, G / H), \mathscr{O}_{G}(\mathscr{V})(-, G / K)\right) .
\end{aligned}
$$


Since, as mentioned at the end of $\S 1$, we can compute homology using cellular chains, it is clear that any realization of $W$ is an equivariant Moore spectrum of type $T$. By $\S 4$, the obstructions to realizing $W$ are the higher order Toda brackets determined by $W$, which is determined by the choice of resolution $P_{*}$. We therefore refer to these as the obstructions to realizing $P_{*}$. Notice also that $\partial^{2}$ is null-homotopic for this tower $W$, so the first possibly nonzero obstruction is $\mu_{2}$.

We can describe the groups in the chain complex ${ }_{p-1} C^{q}$ as

$$
\prod_{n}\left[\Sigma^{p-1} W_{n}, W_{n-q-1}\right]_{G} \cong \prod_{n} \operatorname{Hom}\left(P_{n}, P_{n-q-1} \otimes \omega_{p-1}\right),
$$

where $\omega$ denotes the $\mathscr{O}_{G}(\mathscr{V})$-functor given by equivariant stable homotopy. (The tensor product of $\mathscr{O}_{G}(\mathscr{V})$-functors is defined in the Appendix.) The differential is $\partial^{*}+(-1)^{q} \partial_{*}$, where $\partial$ now refers to the differential of $P_{*}$. The filtration

$$
F_{i}\left(p-1 C^{q}\right)=\prod_{n \geq i} \operatorname{Hom}\left(P_{n}, P_{n-q-1} \otimes \omega_{p-1}\right)
$$

gives rise to a spectral sequence ${ }_{p-1} E$ with

$$
{ }_{p-1} E_{1}^{s, t}=\operatorname{Hom}\left(P_{s}, \operatorname{Tor}_{s-t-1}\left(T, \omega_{p-1}\right)\right),
$$

and

$$
{ }_{p-1} E_{2}^{s, t}=\operatorname{Ext}^{s}\left(T, \operatorname{Tor}_{s-t-1}\left(T, \omega_{p-1}\right)\right) .
$$

This spectral sequence converges to $H^{t}\left(p_{-1} C^{*}, d\right)$ in the sense that the $E_{\infty}$ terms are filtration quotients of $H^{*}\left({ }_{p-1} C^{*}, d\right)$, with $\bigcap_{i} F_{i} H^{*}\left(p-1 C^{*}, d\right)=0$. The following proposition now follows.

Proposition 5.1. If ${ }_{p-1} E_{\infty}^{s, p}=0$ for all $s \geq p+1$, then the obstruction $\mu_{p}$ vanishes. In particular, if $\operatorname{Ext}^{s}\left(T\right.$, $\left.\operatorname{Tor}_{s-p-1}\left(T, \omega_{p-1}\right)\right)=0$ for all $s \geq p+1 \geq 3$, then there exists a connective Moore spectrum $M T$.

The following shows that the realization question is independent of the choice of resolution.

Proposition 5.2. Let $P_{*}$ and $Q_{*}$ be two projective resolutions of $T$. Then we can realize $P_{*}$ iff we can realize $Q_{*}$.

Proof. Choose a map of resolutions $\beta: P_{*} \rightarrow Q_{*}$, and denote the mapping cone of $\beta$ by $R_{*} ; R_{*}$ is a projective resolution of zero since $\beta$ is a chain homotopy equivalence. Now assume that we have a realization of $Q_{*}$ of the form

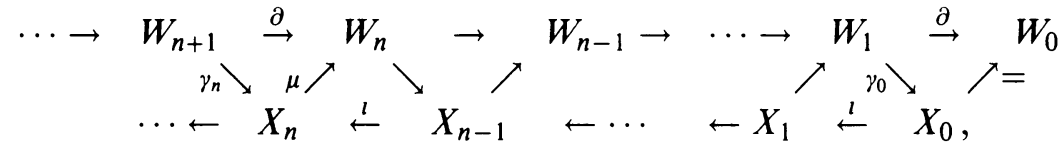

where $\left\{W_{*}, \partial\right\}$ is the tower associated with $Q_{*}$. We assert that there is a realization $Y$ of $R_{*}$ containing $X$ as a subcomplex. Note that this suffices to complete the proof since the desuspension of $Y / X$ will be a realization of $P_{*}$. To prove the assertion, choose a tower $\left\{V_{*}, \partial\right\}$ for $R_{*}$ so that each $W_{n}$ is a wedge summand of $V_{n}$. We now construct inductively the skeleta $Y_{n} \supset X_{n}$ of a realization of $R_{*}$. The start of the induction is obvious in view of the 
equalities $W_{0}=X_{0}$ and $V_{0}=Y_{0}$. For the inductive step, we assume we have a diagram

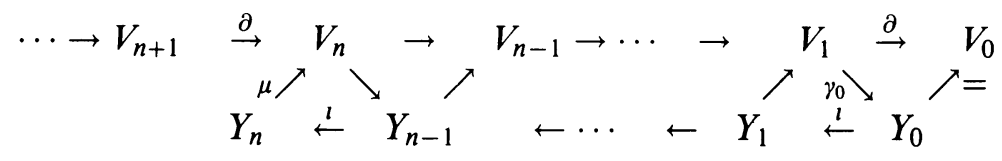

with $Y_{i} \supset X_{i}$ for $i \leq n$. We define a preliminary map $\gamma_{n}^{\prime}: V_{n+1} \rightarrow Y_{n}$ as follows. Since $Y_{n}$ is $(n-1)$-connected, the Hurewicz map $h: \omega_{n}\left(Y_{n}\right) \rightarrow H_{n}\left(Y_{n}\right)$ is an isomorphism, with $H_{n}\left(Y_{n}\right) \cong \operatorname{ker} \partial: R_{n} \rightarrow R_{n-1}$. Since $H_{0}(\partial): H_{0}\left(V_{n+1}\right) \rightarrow$ $H_{0}\left(V_{n}\right)$ has image ker $\partial$, there is a unique factoring of this map through $H_{n}\left(Y_{n}\right)$. By the Hurewicz isomorphism we can now factor $\pi_{0}(\partial): \pi_{0}\left(V_{n+1}\right) \rightarrow \pi_{0}\left(V_{n}\right)$ through $\pi_{0}\left(Y_{n}\right)$, and this determines $\gamma_{n}^{\prime}$ up to homotopy. Since $W_{n+1}$ is a wedge summand of $V_{n+1}$, define $\gamma_{n} \mid W_{n+1}$ as the composite $W_{n+1} \rightarrow X_{n} \hookrightarrow Y_{n}$, and take $\gamma_{n}=\gamma_{n}^{\prime}$ on the other wedge summand. The cofiber $Y_{n+1}$ of $\gamma_{n}$ contains $X_{n+1}$ as a subcomplex.

Note that, in the proof above, $Y$ may be thought of as being obtained from $X$ by attaching cells specified by $P_{*}$ in order to kill off equivariant homotopy groups. In view of the proposition, we may now think of the obstructions $\mu_{p}$ as obstructions to the existence of any equivariant connective Moore spectrum $M T$.

\section{Consequences}

Here we use the theory in $\S 5$, particularly Proposition 5.1 , to give sufficient algebraic conditions for the existence of equivariant Moore spectra. The following result is not surprising, in view of similar results on Moore spaces, such as those in $[\mathrm{K}]$.

Proposition 6.1. If $T$ is any rational $\mathscr{O}_{G}(\mathscr{V})$-functor. Then $M T$ exists.

Proof. By Proposition 5.1, if the $\operatorname{groups}^{\operatorname{Ext}^{s}}\left(T, \operatorname{Tor}_{s-p-1}\left(T, \omega_{p-1}\right)\right)$ vanish for $p>1$, then so do the obstructions to realizing $M T$. Since $\omega_{p-1}$ denotes the $\mathscr{O}_{G}(\mathscr{V})$-functor which assigns to $G / H$ the group $\left[\Sigma^{p} \Sigma^{\infty} G / H^{+}, S\right]_{G}$, where $S$ is the sphere spectrum, it follows that $\omega_{p-1} \otimes \mathbb{Q}=0:$ in the case $\mathscr{V}=\mathbb{R}^{\infty}$ this is the classical result about the finiteness of the nonequivariant stable stems; in the case $\mathscr{V}=\mathscr{U}$ this follows from the splitting of stable equivariant homotopy [tD], which says that $\omega_{p-1}$ is a product of nonequivariant stable homotopy groups of the form $\omega_{p-1}(E W H)$, together with the fact that $E W$ is rationally acyclic for finite groups $W$. It follows that $\operatorname{Tor}_{s-p-1}\left(T, \omega_{p-1}\right)=0$ if $T$ is rational.

The following sharper result for Mackey functors is not unexpected, in view of the folklore belief that inverting the order of the group reduces one to the nonequivariant case. Let $\mathbb{Z}[1 /|G|]$ denote $\mathbb{Z}$ localized away from $|G|$.

Proposition 6.2. If $T$ is any Mackey functor whose underlying groups are finitelygenerated $\mathbb{Z}[1 /|G|]$-modules, then $M T$ exists.

Proof. By Corollary A.5 in the Appendix, $T$ has projective dimension 1 and hence the groups $\operatorname{Ext}^{s}\left(T, \operatorname{Tor}_{s-p-1}\left(T, \omega_{p-1}\right)\right)$ vanish for $s>1$. By Proposition 5.1 , we need only consider $s \geq p+1 \geq 3$, and so the obstructions vanish. 
Let $i: \mathscr{O}_{G}\left(\mathbb{R}^{\infty}\right) \rightarrow \mathscr{O}_{G}(\mathscr{U})$ be the functor induced by the inclusion of $\mathbb{R}^{\infty}$ in $\mathscr{U}$. Then composition $i^{*}$ with $i$ is the map which assigns to each Mackey functor its underlying structure as a Bredon functor. As shown in the Appendix, $i^{*}$ has a left adjoint $i_{*}$.

Proposition 6.3. If $T$ is a Bredon functor of the form $i^{*} T^{\prime}$ for some Mackey functor $T^{\prime}$, and if $T$ has projective dimension $n \leq 2 q-3$ where $q$ is the smallest prime dividing $|G|$, then $M T$ exists.

Proof. The Bredon functor $\omega_{p-1}$ is constant, with value the nonequivariant stable stem, and so $\operatorname{Tor}_{*}\left(T, \omega_{p-1}\right)=\operatorname{Tor}_{*}^{\mathbb{Z}}\left(T, \omega_{p-1}\right)$, the orbitwise Tor with the group $\omega_{p-1}$. Therefore $\operatorname{Tor}_{s-p-1}\left(T, \omega_{p-1}\right)=0$ unless $s-p-1=0$ or 1 , so that either $s=p+1$ or $s=p+2$. If $p-1<2 q-3$, then classical results about nonequivariant stable homotopy say that $\omega_{p-1}$ consists of torsion of order divisible by primes $<q$. Thus $|G|$ is invertible in $\omega_{p-1}$ by definition of $q$. Again using the fact that Tor with $\omega_{p-1}$ is orbitwise Tor with a group, one now has

$$
\begin{aligned}
\operatorname{Ext}^{s}\left(T, \operatorname{Tor}_{s-p-1}\left(T, \omega_{p-1}\right)\right) & =\operatorname{Ext}^{s}\left(T, \operatorname{Tor}_{s-p-1}\left(i^{*} T^{\prime}, \omega_{p-1}\right)\right) \\
& \cong \operatorname{Ext}^{s}\left(T, i^{*} \operatorname{Tor}_{s-p-1}\left(T^{\prime}, \omega_{p-1}\right)\right) \\
& \cong \operatorname{Ext}^{s}\left(i_{*} T, \operatorname{Tor}_{s-p-1}\left(T^{\prime}, \omega_{p-1}\right)\right) \\
& =0
\end{aligned}
$$

by Corollary A.5. On the other hand, if $p-1 \geq 2 q-3$, then $s>2 q-3 \geq$ $\operatorname{dim} T$, so that $\operatorname{Ext}^{s}\left(T, \operatorname{Tor}_{s-p-1}\left(T, \omega_{p-1}\right)\right)=0$, and by Proposition 5.1 we are done.

The stable Steenrod problem is the following: Given a $\mathbb{Z} G$-module $A$, does there exist a $G$-spectrum $M A$ whose nonequivariant homology is $A$ as a $\mathbb{Z} G$ module? If we restrict attention to Bredon spectra $M A$, then the stable Steenrod problem is equivalent to the following: Let $T$ be the Bredon system with $T(G / e)=A$ and $T(G / H)=0$ if $H \neq e$. Does there exist a Bredon spectrum $M T$ ? Indeed, if there is a Bredon spectrum $M A$, then $M A \wedge E G^{+}$is a Moore spectrum of type $T$.

Proposition 6.4. If $A$ is a $\mathbb{Z}[1 /|G|] G$-module, then there exists a connective Bredon spectrum $M A$.

Proof. First observe that the category of $\mathbb{Z} G$-modules is equivalent, via an exact functor $F$, to the full subcategory $\mathscr{E}$ of Bredon functors $T$ with $T(G / H)=0$ unless $H=e$. It follows in particular that projective $\mathbb{Z} G$-modules correspond to the projective Bredon functors in $\mathscr{E}$, so that projective dimension is preserved by $F$. By Lemma A.3, $A$ has projective dimension $\leq 1$, and hence, if $T$ is the corresponding Bredon functor, $\operatorname{Ext}^{s}\left(T, \operatorname{Tor}_{s-p-1}\left(T, \omega_{p-1}\right)\right)=0$ since $s>2$ for the groups relevant to the obstructions.

Proposition 6.5. If $A$ is a $\mathbb{Z} G$-module of projective dimension $n \leq 2 q-3$ where $q$ is the smallest prime dividing $|G|$, then there exists a connective Bredon spectrum $M A$.

Proof. As in Proposition 6.4, the $\mathbb{Z} G$-module $A$ corresponds to a Bredon functor $T$ with $T(G / H)=0$ unless $H=e$. As in the proof of Proposition 6.3, $\operatorname{Ext}^{s}\left(T, \operatorname{Tor}_{s-p-1}\left(T, \omega_{p-1}\right)\right)=0$ unless $s-p-1=0$ or 1 , so that either $s=p+1$ or $s=p+2$. If $p-1<2 q-3$, then again $|G|$ is invertible. Thus 
$\operatorname{Ext}^{s}\left(T, \operatorname{Tor}_{s-p-1}\left(T, \omega_{p-1}\right)\right)=0$ by Lemma A.3. Finally, if $p-1 \geq 2 q-3$, then $s>2 q-3 \geq \operatorname{dim} T$, and so $\operatorname{Ext}^{s}\left(T, \operatorname{Tor}_{s-p-1}\left(T, \omega_{p-1}\right)\right)=0$.

\section{APPENDIX}

Let $\mathscr{S}_{G}(\mathscr{V})$ be a small category of finite $G$-sets, closed under disjoint unions and products, and including a copy of $\mathscr{O}_{G}(\mathscr{V})$. The morphisms $s \rightarrow t$ in $\mathscr{S}_{G}(\mathscr{V})$ are the $\mathscr{V}$-stable $G$-maps $s \rightarrow t$. If $\mathscr{M}_{G}(\mathscr{V})$ denotes the category of $\mathscr{S}_{G}(\mathscr{V})$-functors that preserve direct sums, then $\mathscr{M}_{G}(\mathscr{V})$ is equivalent to the category of $\mathscr{O}_{G}(\mathscr{V})$-functors [L].

The inclusion $i: \mathscr{V} \rightarrow \mathscr{W}$ induces a restriction $i^{*}: \mathscr{M}_{G}(\mathscr{W}) \rightarrow \mathscr{M}_{G}(\mathscr{V})$.

Proposition A.1. $i^{*}$ has a left adjoint $i_{*}: \mathscr{M}_{G}(\mathscr{V}) \rightarrow \mathscr{M}_{G}(\mathscr{W})$.

Proof. Let $T$ be a functor in $\mathscr{M}_{G}(\mathscr{V})$. The required left adjoint is given by

$$
i_{*}(T)(s)=\sum_{s \rightarrow t} T(t) / \sim
$$

the sum running over maps in $\mathscr{O}_{G}(\mathscr{W})$, and the identification being given by

$$
(x)_{s \rightarrow t \stackrel{f}{\rightarrow} u} \sim\left(f^{*} x\right)_{s \rightarrow t}
$$

for any map $f$ in $\mathscr{O}_{G}(\mathscr{V})$.

Note that $i_{*}$ preserves the canonical projectives; that is $i_{*} \mathscr{S}_{G}(\mathscr{V})(-, a)=$ $\mathscr{S}_{G}(\mathscr{W})(-, a)$. It follows that

$$
\operatorname{Ext}^{*}\left(S, i^{*} T\right) \cong \operatorname{Ext}^{*}\left(i_{*} S, T\right) .
$$

The category $\mathscr{M}_{G}(\mathscr{V})$ possesses an internal Hom functor given by

$$
\operatorname{Hom}(S, T)(a)=\mathscr{M}_{G}(\mathscr{V})\left(S, T_{a}\right)
$$

where $T_{a}(b)=T(a \times b)$.

Proposition A.2. $\mathscr{M}_{G}(\mathscr{V})$ has an internal tensor product $\otimes$ such that

$$
\operatorname{Hom}(S \otimes T, U) \cong \operatorname{Hom}(S, \operatorname{Hom}(T, U)) .
$$

Proof. We construct the tensor product explicitly by taking

$$
(S \otimes T)(a)=\sum_{a \rightarrow b \times c} S(b) \otimes T(c) / \sim
$$

where the identification is given by

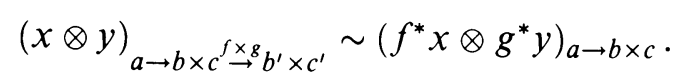

Note that the above construction behaves correctly on canonical projectives, namely $\mathscr{S}_{G}(\mathscr{V})(-, a) \otimes \mathscr{S}_{G}(\mathscr{V})(-, b) \cong \mathscr{S}_{G}(\mathscr{V})(-, a \times b)$. This implies that the equivariant cellular chain complex of a smash product of equivariant $\mathrm{CW}$ spectra is the tensor product of the cellular chain complexes of the spectra.

If we invert $|G|$, then we have the following well-known fact about $\mathbb{Z} G$ modules: 
Lemma A.3. Away from $|G|$, every finitely generated $\mathbb{Z} G$-module has projective length $\leq 1$.

Proof. It suffices to show that every finitely generated torsion-free $\mathbb{Z} G$-module is projective. Since such a module is projective as a $\mathbb{Z}$-module, one can average over $G$ to produce $\mathbb{Z} G$-lifts.

It is also well known (cf. [tD, LMS]) that, with $|G|$ inverted, the Burnside ring $A(G)$ is isomorphic to $\Pi_{(H)} \mathbb{Z}[1 /|G|]$, a product of copies of $\mathbb{Z}[1 /|G|]$, one for each conjugacy class of subgroups of $G$. This splitting comes about because of the presence of primitive idempotents $e_{H} \in A(G)[1 /|G|]$, one for each conjugacy class. Since any Mackey functor $T$ is a module over $A(G)$ (in the sense that $A(G)$ acts on each level so that the Mackey functor structure maps are $A(G)$-module homomorphisms) it follows that $T$ splits into corresponding summands, which we identify in the following result.

Proposition A.4. Let $T$ be a Mackey functor. Then, away from $|G|$,

$$
T \cong \sum_{(H)} e_{H} T \cong \sum_{(H)}\left(e_{H} A_{G / H} \otimes_{\mathbb{Z}} e_{H} T(G / H)\right)^{W H},
$$

where $A_{G / H}(s)=\mathscr{S}_{G}(\mathscr{U})(G / H, s)$ and $W H=N H / H$ acts diagonally.

Proof. First observe that there is an isomorphism

$$
e_{H} A_{G / H}(s) \cong \mathbb{Z}[1 /|G|](G / H, s),
$$

where the latter is the free $\mathbb{Z}[1 /|G|]$-module on the set of $G$-maps $G / H \rightarrow s$. The isomorphism is given by observing that $e_{H}$ annihilates stable maps of the form $G / H \leftarrow G / K \rightarrow s$ for $(K)<(H)$, leaving only the morphisms corresponding to sums of actual $G$-maps. We define a map $\kappa: e_{H} \mathbb{Z}[1 /|G|](G / H, s)$ $\otimes_{\mathbb{Z}} e_{H} T(G / H) \rightarrow e_{H} T(s)$ by $\kappa(f \otimes t)=f_{*}(t)$; this is natural in $s$. Also define $\lambda: e_{H} T(s) \rightarrow\left(e_{H} \mathbb{Z}[1 /|G|](G / H, s) \otimes_{\mathbb{Z}} e_{H} T(G / H)\right)^{W H}$ by

$$
\lambda(t)=\sum_{f: G / H \rightarrow s} f \otimes f^{*}(t) .
$$

If we restrict $\kappa$ to the $W H$-fixed set, then it is tedious but straightforward to see that $\kappa \lambda$ and $\lambda \kappa$ are both automorphisms. Therefore $\kappa$ is an isomorphism.

Corollary A.5. If, as a $\mathbb{Z}[1 /|G|]$-module, each $T(G / H)$ is finitely generated, then $T$ has projective length $\leq 1$.

Proof. In the splitting

$$
T \cong \sum_{(H)}\left(e_{H} A_{G / H} \otimes_{\mathbb{Z}} e_{H} T(G / H)\right)^{W H},
$$

each $e_{H} A_{G / H}$ is projective as a Mackey functor and the group $e_{H} T(G / H)$ has projective length $\leq 1$ as a $W H$-module, by Lemma A.3. If we take a resolution of length two of $e_{H} T(G / H)$ by projective $W H$-modules and tensor with $e_{H} A_{G / H}$, then we get a projective resolution of $e_{H} A_{G / H} \otimes_{\mathbb{Z}} e_{H} T(G / H)$ of length two. Since we are away from $|G|$, taking $W H$-fixed sets is multiplication by an idempotent in $\mathbb{Z}[1 /|G|] W H$, and hence exact. Passage to fixed-sets therefore gives us a projective resolution of $T$ of length 2 . 


\section{REFERENCES}

[B] G. E. Bredon, Equivariant cohomology theories, Lecture Notes in Math., vol. 34, SpringerVerlag, 1967.

[C1] G. Carlsson, A counterexample to a conjecture of Steenrod, Invent. Math. 64 (1981), 171174.

[C2] J. M. Cohen, The decomposition of stable homotopy, Ann. of Math. 87 (1968), 305-320.

[CW] S. R. Costenoble and S. Waner, A nonexistence result for Moore G-spectra, Proc. Amer. Math. Soc. 113 (1991), 265-274.

[tD] T. tom Dieck, Transformation groups and representation theory, Lecture Notes in Math., vol. 766, Springer-Verlag, 1979.

[K] P. J. Kahn, Rational Moore G-spaces, Trans. Amer. Math. Soc. 298 (1986), 245-271.

[L] L. G. Lewis, Jr., Mimeographed notes, Syracuse University.

[LMS] L. G. Lewis, Jr., J. P. May, and M. Steinberger, Equivariant stable homotopy theory, Lecture Notes in Math., vol. 1213, Springer-Verlag, 1986.

[M] S. Mac Lane, Categories for the working mathematician, Springer-Verlag, 1971.

[Q] F. Quinn, Finite nilpotent group actions on finite complexes, Geometric Applications of Homotopy Theory. I, Lecture Notes in Math., vol. 657, Springer-Verlag, 1978, pp. 375407.

[S1] J. R. Smith, Topological realization of chain complexes 1. The general theory, Topology Appl. 22 (1986), 301-313.

[S2] E. Spanier, Higher order operations, Trans. Amer. Math. Soc. 109 (1963), 509-539.

[W] S. Waner, Mackey functors and G-cohomology, Proc. Amer. Math. Soc. 90 (1984), 641-648.

Department of Mathematics, Hofstra University, Hempstead, New York 11550

E-mail address: matsrc@vaxc.hofstra.edu 